\title{
Could Mean Platelet Volume Be a Reliable Indicator for Acute Mesenteric Ischemia Diagnosis? A Case-Control Study
}

\author{
Vermi Degerli, ${ }^{1}$ Isil Ergin,, ${ }^{2}$ Fulya Yilmaz Duran, ${ }^{3}$ \\ Mehmet Akif Ustuner, ${ }^{4}$ and Ozgur Duran ${ }^{1}$ \\ ${ }^{1}$ Department of Emergency Medicine, Izmir Bozyaka Training and Research Hospital, Bozyaka, Izmir, Turkey \\ ${ }^{2}$ Department of Public Health, Ege University School of Medicine, Bornova, Izmir, Turkey \\ ${ }^{3}$ Department of Anaesthesiology and Reanimation, Izmir Bozyaka Training and Research Hospital, Bozyaka, Izmir, Turkey \\ ${ }^{4}$ Department of Surgery, Dr. Abdurrahman Yurtaslan Ankara Oncology Training and Research Hospital, Yenimahalle, \\ 06200 Ankara, Turkey
}

Correspondence should be addressed to Fulya Yilmaz Duran; drfulya@mynet.com

Received 18 May 2016; Revised 1 August 2016; Accepted 14 August 2016

Academic Editor: Demosthenes Bouros

Copyright (C) 2016 Vermi Degerli et al. This is an open access article distributed under the Creative Commons Attribution License, which permits unrestricted use, distribution, and reproduction in any medium, provided the original work is properly cited.

Objective. Acute mesenteric ischemia (AMI) is a disease, usually seen in elderly people and accompanied by comorbid diseases. Mean platelet volume (MPV), the significant indicator of platelet activation and function, is associated with AMI. In this study, we considered that we can use MPV as a reliable indicator in the diagnosis of AMI. Methods. This study was conducted among AMI patients with two control groups. Age, gender, MPV, platelet count, concomitant diseases, abdominal computed tomography, and patient outcomes were recorded for evaluation. Control group I contained 41 healthy patients whose ages-genders were matched. Control group II contained 41 patients with no AMI, whose ages-genders-concomitant diseases were matched. Results. Of the total 41 AMI patients, 22 were female and 19 were male. The average age of them was $72.12 \pm 13.2$ (44-91) years. MPV was significantly increased in the AMI $(p=0.001)$ and control group II $(p<0.001)$ in comparison with healthy control groups. In the comparison of the AMI patients with their matched controls for concomitant diseases, no statistical difference was found in the MPV values. Conclusion. MPV may be used as an indicator of AMI only if the patient has no concomitant diseases. The existence of a concomitant disease brings into question the reliability of high MPV values as a suitable indicator.

\section{Introduction}

Acute mesenteric ischemia (AMI) is a rare disease, and it accounts for approximately $0.1 \%$ of patients getting treatment in emergency department [1-3]. The causes of AMI are embolism (50\%), thrombosis (20\%), nonocclusive factors (20\%), and venous thrombosis (10\%). The most common risk factors are age, myocardial infarction, dysrhythmias (especially atrial fibrillation), atherosclerosis, cardiac failure, hypercoagulability, intra-abdominal trauma or infection, and malignancy $[1,2,4]$. Early diagnosis and surgical intervention are very important for reducing mortality. Yet, the AMI diagnosis is difficult, because clinical signs are nonspecific, and there is no exact laboratory marker for AMI. Gold standard for the diagnosis of AMI is multidetect row computed tomography. Serum plasma markers such as leukocytes, Ddimer, ischemia-modified albumin, urinary and plasma fatty acid-binding proteins, and serum lactate levels do not have sufficient diagnostic accuracy for the diagnosis of AMI $[1,3]$.

Platelet aggregation and coagulation activation are fundamental for clot formation in the arterial and venous thrombosis [5, 6]. AMI is seen in both arterial and venous system; the origin is $80 \%$ thromboembolic disease. Due to an increase in thrombogenic activity, an increase in MPV is to be expected. Platelet volume is a marker of platelet function and activation that is readily measured as MPV. It can be routinely measured by automated cell counter. It is a simple parameter inexpensive to obtain and easy to interpret. Circulating platelets may differ according to their density, reactivity, and size. The larger platelets contain more dense 
granules. They are metabolically and enzymatically more active than smaller ones. They have more thrombotic and inflammatory property [5-12].

There is only a few studies evaluating the role of MPV in AMI patients [12-14]. Our research questions are as follows: (i) Does MPV increase in AMI patients in comparison with healthy controls? (ii) Does MPV increase in patients with concomitant diseases in comparison with healthy controls? (iii) Can the MPV increase be attributed to AMI only?

\section{Materials and Methods}

This study was conducted at emergency department of our hospital between January 2008 and December 2014. After ethical committee approval was obtained, all baseline data were collected retrospectively from patients' medical records. The data consisted of demographics, concomitant diseases, laboratory tests including complete blood count, imaging tests including abdominal computed tomography, and patient outcomes. Patients with history of malignancy, chronic hematological diseases, autoimmune disease, hepatic dysfunction, acute or chronic inflammatory disease, pregnancy, and cranial trauma were excluded. The outcomes of the cases were grouped as survival or exitus from the records.

Regarding effect size as 0.55 and $\alpha$ error probability 0.05 , the power of this study is $80 \%$ for 41 cases and 41 controls.

The case group of the study was comprised of AMI patients. Hospital records of 41 patients (age $\geq 18$ years) who were operated on with the diagnosis of AMI and pathological specimens revealed AMI were reviewed retrospectively. The study included two control groups: control group I: age-sexmatched 41 healthy patients with no concomitant diseases; control group II: age-sex-concomitant disease-matched 41 patients who were not diagnosed for mesenteric ischemia.

During admission to the emergency department, patients peripheral venous blood samples were drawn by careful vein puncture in $20 \mathrm{G}$ sterile syringe. For measurement of MPV and platelet count (PC), $2 \mathrm{~mL}$ of blood was drawn into a vacutainer tube, containing EDTA as an anticoagulant and analyzed within 60 minutes in an automated blood cell counter (BC-6800 analyzer, Mindray, China). Normal range for MPV was 7-10.7 femtoliter (fL) in our center laboratory.

2.1. Statistical Analysis. Statistical analysis was performed using SPSS 20.0 for Windows (SPSS, Chicago, IL, USA). The results were reported as mean \pm standard deviation (SD) for the quantitative variables and percentages for the categorical variables. The groups with the different MPV counts and PC were compared by using Student's $t$-test and its nonparametric variant Mann-Whitney $U$ (MWU) as appropriate. The Pearson test was used for correlation of MPV and PCs. Statistical significance was defined as $p$ values less than 0.05 .

Comparisons were made for (1) AMI and control group I, (2) AMI and control group II, and (3) control group I and control group II.

In the receiver operating characteristic (ROC) curve analysis, only the control group I has been compared with AMI group. This comparison has been chosen because the
TABLE 1: Demographic and clinical characteristics of the mesenteric ischemia patients.

\begin{tabular}{lc}
\hline Characteristics & $\begin{array}{c}\text { Mesenteric } \\
\text { ischemia group } \\
(n=41)\end{array}$ \\
\hline Age (years \pm SD) & $72.12 \pm 13.2$ \\
Female, $n(\%)$ & $22(53.7)$ \\
Computed abdomen tomography & $25(86.2)^{*}$ \\
positive, $n(\%)$ & $23(57.5)^{* *}$ \\
Outcome patient/exitus, $n(\%)$ & $25(61.0)$ \\
Hypertension, $n(\%)$ & $20(48.8)$ \\
Coronary artery accident, $n(\%)$ & $14(34.1)$ \\
Diabetes mellitus, $n(\%)$ & $9(22.0)$ \\
Atrial fibrillation, $n(\%)$ & $5(12.2)$ \\
Heart failure, $n$ (\%) & $5(12.2)$ \\
Cerebrovascular disease, $n(\%)$ & $4(9.8)$ \\
Chronic renal failure, $n(\%)$ & $3(7.3)$ \\
Acute renal failure, $n(\%)$ & $3(7.3)$ \\
Chronic obstructive pulmonary disease, $n$ & $1(2.4)$ \\
E) & $1(2.4)$ \\
Epilepsy, $n$ (\%) & \\
Deep vein thrombosis, $n(\%)$ &
\end{tabular}

${ }^{*}$ Among 29 patients who had CT.

**A Among 40 patients treated.

comparison between all non-AMI patients and AMI patients resulted in a ROC curve with area under the curve $=0.575$ (SE $=0.055, p=0.176$ ), while the comparison between control group I and AMI resulted in a better ROC curve with area under the curve $=0.690(\mathrm{SE}=0.059, p=0.003)$. To calculate the best cut-off in ROC curve, Youden's index was used in which "sensitivity + specificity - 1" was maximal.

\section{Results}

Among the patients with AMI, there were 22 females (53.7\%) and 19 males (46.3\%); the average age of them was $72.12 \pm$ 13.2 (44-91) years. All patients had a history of concomitant disease; and the four diseases following were hypertension, cardiovascular disorders, diabetes mellitus, and atrial fibrillation, consecutively. Baseline characteristics for AMI are summarized in Table 1. Control I and control II groups' average ages were $72.34 \pm 13.0(45-91)$ and $72.21 \pm 13.4$ (4190) years, respectively.

Table 2 shows the laboratory characteristics of case and control groups. While PC does not show any significant difference in the comparison of three groups, MPV is significantly increased in the ischemia $(p=0.001)$ and concomitant disease group $(p<0.001)$ in comparison with the healthy group. However, no significant difference was found between the ischemia and the concomitant disease group $(p=0.563)$.

In Table 3, the results of the MPV comparisons for concomitant disease groups with (cases) and without (control group II) AMI are presented. In the comparison of the AMI patients with their matched controls for concomitant 
TABLE 2: Laboratory characteristics of AMI group and control groups.

\begin{tabular}{|c|c|c|c|c|c|}
\hline Variables & AMI group $(n=41)$ & Control group I* $(n=41)$ & $p$ & Control group $\mathrm{II}^{* *}(n=41)$ & $p$ \\
\hline $\mathrm{PC}\left(\times 10^{3} / \mathrm{mL}\right)$ & $232.05 \pm 86.26$ & $252.51 \pm 60.10$ & 0.216 & $264.85 \pm 73.81$ & 0.068 \\
\hline MPV (fL) & $9.65 \pm 1.31$ & $8.79 \pm 0.80$ & 0.001 & $9.83 \pm 1.47$ & 0.563 \\
\hline
\end{tabular}

AMI, acute mesenteric ischemia; PC, platelet count; MPV, mean platelet volume.

*Age- and sex-matched from healthy 41 patients.

**Age, sex, and concomitant disease-matched from 41 patients, but no mesenteric ischemia.

TABLE 3: The results of the MPV comparisons of concomitant disease groups with (cases) and without (control group II) AMI.

\begin{tabular}{|c|c|c|c|c|}
\hline Concomitant disease & $\begin{array}{l}\text { AMI group } \\
\quad(n=41)\end{array}$ & $\begin{array}{c}\text { Control group II } \\
(n=41)\end{array}$ & $\begin{array}{c}\text { MPV } \\
\text { MWU* }^{*}\end{array}$ & $\begin{array}{c}\mathrm{MPV} \\
p\end{array}$ \\
\hline Hypertension, $n$ & 25 & 26 & 305.000 & 0.706 \\
\hline Coronary artery accident, $n$ & 20 & 20 & 159.000 & 0.267 \\
\hline Diabetes mellitus, $n$ & 14 & 14 & 71.000 & 0.214 \\
\hline Atrial fibrillation, $n$ & 9 & 8 & 23.000 & 0.210 \\
\hline Heart failure, $n$ & 5 & 4 & 4.000 & 0.142 \\
\hline Cerebrovascular disease, $n$ & 5 & 5 & 6.500 & 0.209 \\
\hline Chronic renal failure, $n$ & 4 & 2 & 4000 & 1.000 \\
\hline Acute renal failure, $n$ & 3 & 1 & 0.0 & 0.180 \\
\hline Chronic obstructive pulmonary disease, $n$ & 3 & 3 & 0.0 & 0.100 \\
\hline Epilepsy, $n$ & 1 & 1 & 0.0 & 0.317 \\
\hline Deep vein thrombosis, $n$ & 1 & 1 & 0.0 & 0.317 \\
\hline
\end{tabular}

${ }^{*}$ Mann-Whitney $U$.

diseases, no statistical difference was founded in the MPV values (Table $3, p>0.05$ ).

In the comparison of the outcomes (survival/exitus) of mesenteric ischemia, there was no significant difference in the MPV $(M W U=193.5, p=0.956)$ and PC $(M W U=134.5$, $p=0.095)$. In the AMI patients, control I and control II groups, there was seen a low level of negative correlation in PC and MPV, significantly. The Pearson correlation coefficients in each group were $0.364,0.361$, and 0.334 , respectively $(p<0.05)$.

ROC curve analysis suggested that the best MPV value cut-off point for AMI was $8.6 \mathrm{fL}$, sensitivity was $70 \%$, and specificity was $53 \%$ (area under the curve $=0.690$ ) (Figure 1 ). Positive likelihood ratio was 1.52 and negative likelihood ratio was 1.83 for this cut-off.

\section{Discussion}

This study is a first attempt to evaluate the MPV value among AMI patients in comparison with two controls: healthy and concomitant disease with no AMI. An important finding is obtained from this study that reliance on high MPV values in diagnosis of AMI may be insufficient in the existence of concomitant diseases.

When we examine the literature, Altintoprak et al. are the first to investigate the relationship between MPV and AMI. In their study they reported that MPV values were higher in nonsurvivors $(n=15, \mathrm{MPV}=9.01)$ than in survivors $(n=$ $15, \mathrm{MPV}=7.8)$ [14]. Bilgiç et al. investigated whether MPV was associated with outcome of AMI and reported that MPV values were higher in nonsurvivors $(n=35, \mathrm{MPV}=8.4 \mathrm{fL})$

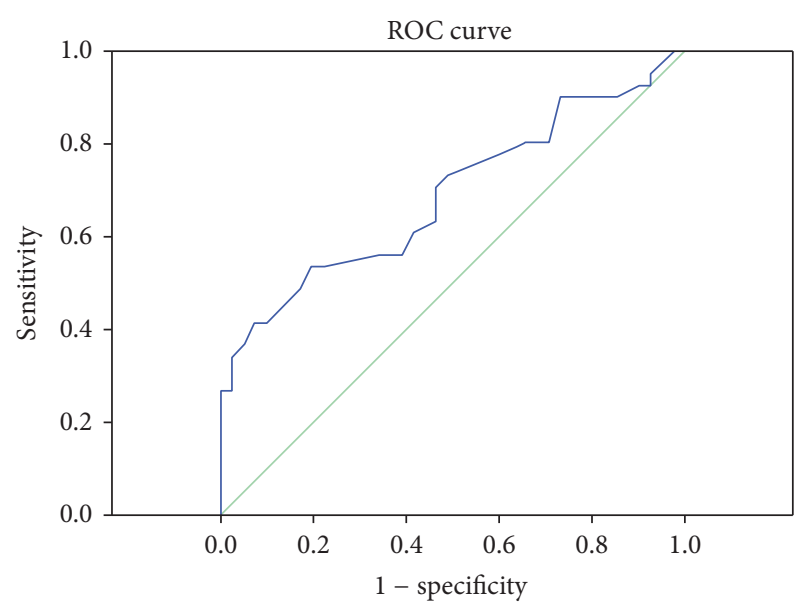

FIGURE 1: Receiver operating characteristic curve results for mean platelet volume in patients with acute mesenteric ischemia.

than in survivors $(n=26, \mathrm{MPV}=7.6 \mathrm{fL})$ [12]. In our study, it is revealed that MPV was significantly higher in patients with AMI compared to healthy controls ( $9.6 \mathrm{fL}$ and $8.7 \mathrm{fL}$, resp.). In a recent study, Türkoğlu et al. reported that MPV was higher in patients with AMI compared to healthy volunteers (9.4 fL and 7.4 fL, resp.) [13]. MPV is a simple parameter that indicates the platelet size. When compared with small ones, large platelets are more prone to aggregation and inflammation, and they produce prothrombotic substances. In addition, large platelets are less sensitive to the inhibitory effect of prostacyclin on aggregation and secretion compared 
to small ones [5]. Therefore, higher MPV values in patients with AMI can be explained by inflammatory and thrombotic features of the disease.

To our knowledge, there is no study in the literature that evaluates if higher MPV values in patients with AMI arise from ischemia or concomitant diseases of these patients. Previous studies have reported that there is increase in MPV in patients with coronary artery disease $[7,11,15$, 16], hypertension $[8,9,17]$, diabetes mellitus [11, 18], atrial fibrillation [11], congestive heart failure [7], acute ischemic brain stroke $[10,11,19-21]$, and venous thromboembolism $[6,22]$. However, some authors have revealed contrasting data as well [23-26].

According to our study results, when compared with healthy controls, MPV was significantly higher in patients without diagnosis of AMI but with concomitant diseases (control group II). Regarding this finding, the MPV level of the patients with AMI may be confounded by their concomitant diseases. In the study herein, most of the AMI patients were geriatric, and they had concomitant diseases. So, we presume that MPV might be higher in these patients before the diagnosis of AMI. The elevated MPV in AMI, when adjusted for concomitant diseases, appears related to concomitant diseases rather than AMI.

We also reported that MPV value and $\mathrm{PC}$ are not associated with the mortality of the AMI patients. Altintoprak et al. and Bilgiç et al. disclosed that there are higher MPV values in the exitus group compared with the survival group in patients with AMI $[12,14]$. They revealed that higher MPV values are poor prognostic parameters among patients with AMI. Our study results do not show higher MPV associated with mortality. This can be explained with the high age profile of our AMI patient group compared with the studies revealed.

We determine a negative correlation between MPV values and PC parallel to findings of previous studies. Although the relationship between MPV and PC is not fully understood, some studies reported that increase in volumes of platelets is associated with decrease in platelet count. Under normal conditions, the circulating platelet mass (platelet count $\times$ $\mathrm{MPV})$ is kept constant $[5,6,9,11]$.

In our study the cut-off value for MPV was determined to be $8.6 \mathrm{fL}$ with $70 \%$ sensitivity and $53 \%$ specificity. While Bilgiç et al. reported MPV level cut-off points for AMI 8.1 fL with $60 \%$ sensitivity and $73 \%$ specificity, Türkoğlu et al. reported MPV level cut-off points for AMI 8.1 fL with 83.2\% sensitivity and $80 \%$ specificity $[12,13]$. Patients diagnosed with AMI are geriatric and often have concomitant diseases. MPV values are reported higher especially in cardiovascularrelated comorbid diseases [6-11, 15-22]. According to our study results, higher MPV values were reported in patients with diagnosis of AMI who have concomitant diseases and in patients without diagnosis of AMI but with concomitant diseases (control group II). Thus we can explain high MPV values not only by AMI but also with concomitant diseases. Regarding this finding, we can explain lower sensitivity and specificity values in our study.

There are some limitations in this study. We neglected the drugs used by the patients in our study group. In a few studies, it is found that antiplatelet drugs and lipid-lowering drugs have effects on platelet size; but the results are controversial $[5,7,20]$. The study is a retrospective evaluation limited with information in the patient data

\section{Conclusions}

MPV may be used as an indicator of AMI only if the patient has no concomitant diseases. The existence of a concomitant disease brings questions about the reliance of high MPV values as a good indicator in diagnosis of AMI. Executing investigations in a prospective study designed with larger patient groups may bring more insight to the current findings.

\section{Ethical Approval}

Ethics committee approval was received for this study from the ethics committee of "X Training and Research Hospital."

\section{Competing Interests}

The authors declared no competing interests related to this paper.

\section{Authors' Contributions}

Vermi Degerli and Isil Ergin conceived the study. Vermi Degerli organized the program design and wrote the initial draft of the manuscript. Isil Ergin performed the statistical analyses. Vermi Degerli, Mehmet Akif Ustuner, Fulya Yilmaz Duran, and Ozgur Duran contributed to data collection. Vermi Degerli, Isil Ergin, Fulya Yilmaz Duran, Mehmet Akif Ustuner, and Ozgur Duran reviewed and participated in manuscript revisions and all authors approved the final version submitted. Vermi Degerli takes responsibility for the paper as a whole.

\section{References}

[1] T. C. van den Heijkant, B. A. C. Aerts, J. A. Teijink, W. A. Buurman, and M. D. P. Luyer, "Challenges in diagnosing mesenteric ischemia," World Journal of Gastroenterology, vol. 19, no. 9, pp. 1338-1341, 2013.

[2] J. P. Martinez and G. J. Hogan, "Mesenteric ischemia," Emergency Medicine Clinics of North America, vol. 22, no. 4, pp. 909$928,2004$.

[3] M. T. Cudnik, S. Darbha, J. Jones, J. Macedo, S. W. Stockton, and B. C. Hiestand, "The diagnosis of acute mesenteric ischemia: a systematic review and meta-analysis," Academic Emergency Medicine, vol. 20, no. 11, pp. 1087-1100, 2013.

[4] J. L. Bobadilla, "Mesenteric ischemia," Surgical Clinics of North America, vol. 93, no. 4, pp. 925-940, 2013.

[5] A. Y. Gasparyan, L. Ayvazyan, D. P. Mikhailidis, and G. D. Kitas, "Mean platelet volume: a link between thrombosis and inflammation?" Current Pharmaceutical Design, vol. 17, no. 1, pp. 47-58, 2011.

[6] S. K. Brækkan, E. B. Mathiesen, I. Njølstad, T. Wilsgaard, J. StøRmer, and J. B. Hansen, "Mean platelet volume is a risk factor for venous thromboembolism: the Tromsø study," Journal of Thrombosis and Haemostasis, vol. 8, no. 1, pp. 157-162, 2010. 
[7] N. Kilicli-Camur, R. Demirtunc, C. Konuralp, A. Eskiser, and Y. Basaran, "Could mean platelet volume be a predictive marker for acute myocardial infarction?" Medical Science Monitor, vol. 11, pp. CR387-CR392, 2005.

[8] M. Karabacak, A. Dogan, A. K. Turkdogan, M. Kapci, A. Duman, and O. Akpinar, "Mean platelet volume is increased in patients with hypertensive crises," Platelets, vol. 25, no. 6, pp. 423-426, 2014.

[9] S. G. Chu, R. C. Becker, P. B. Berger et al., "Mean platelet volume as a predictor of cardiovascular risk: a systematic review and meta-analysis," Journal of Thrombosis and Haemostasis, vol. 8, no. 1, pp. 148-156, 2010.

[10] F. Ghahremanfard, N. Asghari, R. Ghorbani, A. Samaei, H. Ghomi, and M. Tamadon, "The relationship between mean platelet volume and severity of acute ischemic brain stroke," Neurosciences, vol. 18, no. 2, pp. 147-151, 2013.

[11] L. Vizioli, S. Muscari, and A. Muscari, "The relationship of mean platelet volume with the risk and prognosis of cardiovascular diseases," International Journal of Clinical Practice, vol. 63, no. 10, pp. 1509-1515, 2009.

[12] İ. C. Bilgiç, S. Gelecek, M. M. Ozmen, and B. Kasapoglu, "The association of elevated mean platelet volume with the outcome of acute mesenteric ischemia," Blood Coagulation \& Fibrinolysis, vol. 26, no. 7, pp. 727-730, 2015.

[13] A. Türkoğlu, M. Gül, A. Oğuz et al., "Mean platelet volume: is it a predictive parameter in diagnosis of acute mesenteric ischemia?" International Surgery, vol. 100, no. 5, pp. 962-965, 2015.

[14] F. Altintoprak, Y. Arslan, O. Yalkin, Y. Uzunoglu, and O. V. Ozkan, "Mean platelet volume as a potential prognostic marker in patients with acute mesenteric ischemia-retrospective study," World Journal of Emergency Surgery, vol. 8, article 49, 2013.

[15] H. Şenaran, M. Ileri, A. Altinbaş et al., "Thrombopoietin and mean platelet volume in coronary artery disease," Clinical Cardiology, vol. 24, no. 5, pp. 405-408, 2001.

[16] G. Endler, A. Klimesch, H. Sunder-Plassmann et al., "Mean platelet volume is an independent risk factor for myocardial infarction but not for coronary artery disease," British Journal of Haematology, vol. 117, no. 2, pp. 399-404, 2002.

[17] S. Nadar, A. D. Blann, and G. Y. H. Lip, "Platelet morphology and plasma indices of platelet activation in essential hypertension: effects of amlodipine-based antihypertensive therapy," Annals of Medicine, vol. 36, no. 7, pp. 552-557, 2004.

[18] N. Papanas, G. Symeonidis, E. Maltezos et al., "Mean platelet volume in patients with type 2 diabetes mellitus," Platelets, vol. 15, no. 8, pp. 475-478, 2004.

[19] F. Mayda-Domaç, H. Misirli, and M. Yilmaz, "Prognostic role of mean platelet volume and platelet count in ischemic and hemorrhagic stroke," Journal of Stroke and Cerebrovascular Diseases, vol. 19, no. 1, pp. 66-72, 2010.

[20] S. Greisenegger, G. Endler, K. Hsieh, S. Tentschert, C. Mannhalter, and W. Lalouschek, "Is elevated mean platelet volume associated with a worse outcome in patients with acute ischemic cerebrovascular events?” Stroke, vol. 35, no. 7, pp. 1688-1691, 2004.

[21] P. Bath, C. Algert, N. Chapman, and B. Neal, "Association of mean platelet volume with risk of stroke among 3134 individuals with history of cerebrovascular disease," Stroke, vol. 35, no. 3, pp. 622-626, 2004.
[22] M. Gulcan, E. Varol, M. Etli, F. Aksoy, and M. Kayan, "Mean platelet volume is increased in patients with deep vein thrombosis," Clinical and Applied Thrombosis/Hemostasis, vol. 18, no. 4, pp. 427-430, 2012.

[23] P. M. W. Bath, C. Carney, N. D. Markandu, and G. A. MacGregor, "Platelet volume is not increased in essential hypertension," Journal of Human Hypertension, vol. 8, no. 6, pp. 457-459, 1994.

[24] S. Damodar, K. V. Ganesh, and S. Murthy, "Mean platelet volume does not predict risk of myocardial infarction or coronary artery disease in Indian patients," Platelets, vol. 19, no. 1, pp. 80-81, 2008.

[25] T. O'Malley, P. Langhorne, R. A. Elton, and C. Stewart, "Platelet size in stroke patients," Stroke, vol. 26, no. 6, pp. 995-999, 1995.

[26] E. D’Erasmo, G. Aliberti, F. S. Celi, E. Romagnoli, E. Vecci, and G. F. Mazzuoli, "Platelet count, mean platelet volume and their relation to prognosis in cerebral infarction," Journal of Internal Medicine, vol. 227, no. 1, pp. 11-14, 1990. 


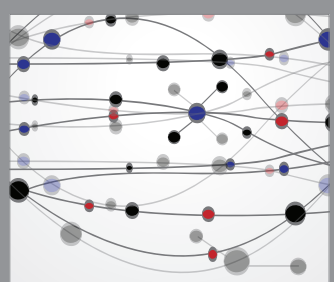

The Scientific World Journal
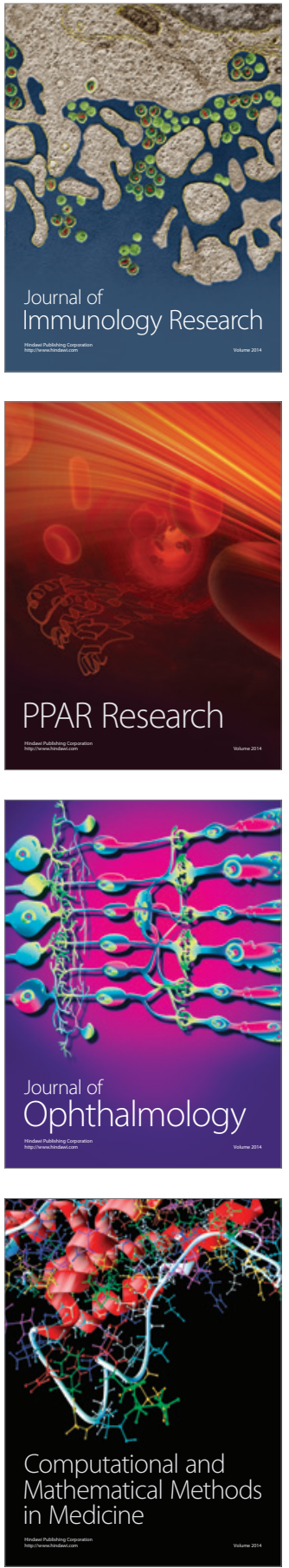

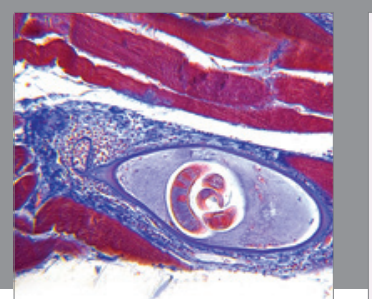

Gastroenterology Research and Practice

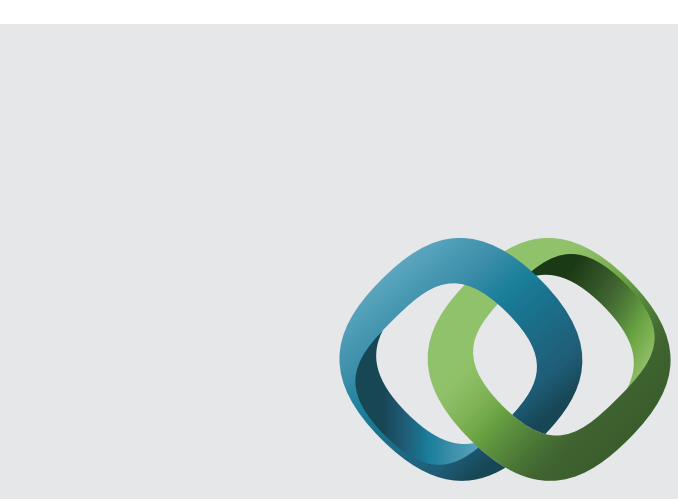

\section{Hindawi}

Submit your manuscripts at

http://www.hindawi.com
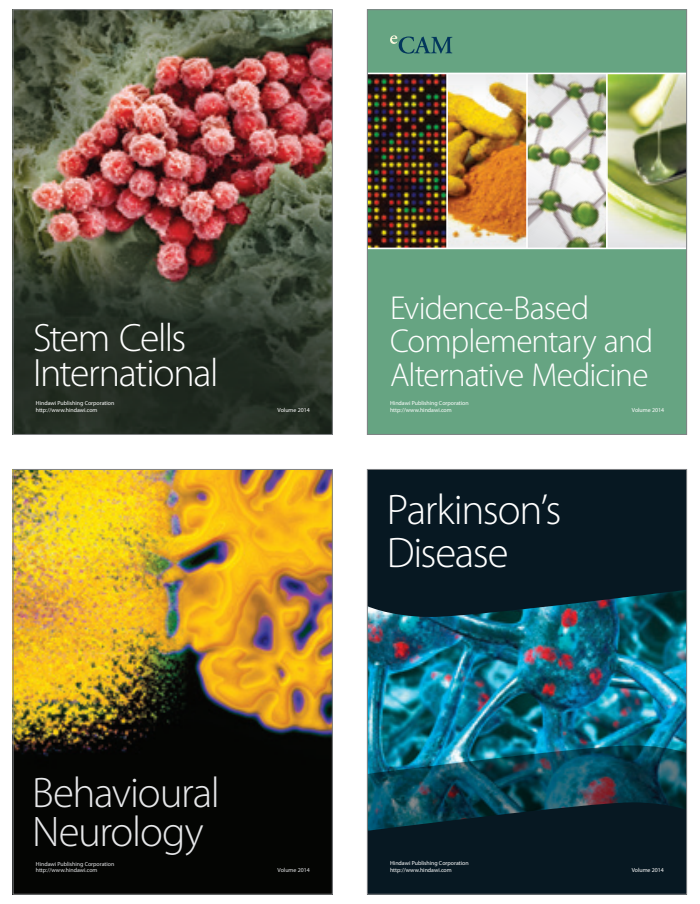
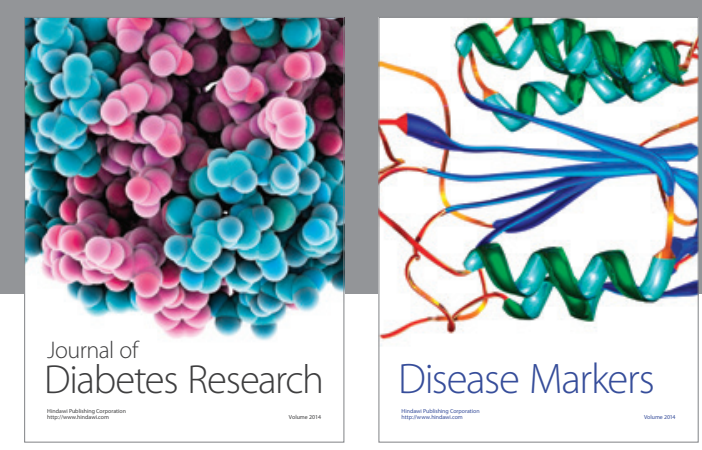

Disease Markers
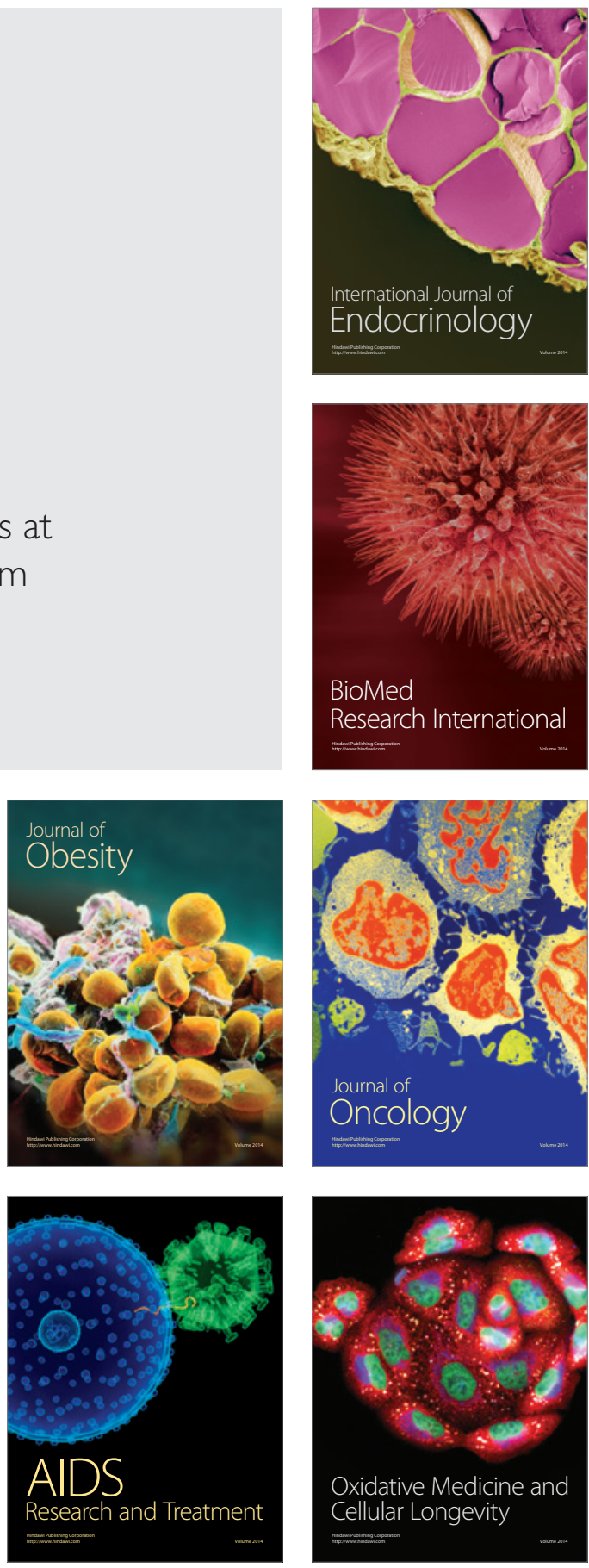